\title{
Valoración y diagnóstico del síndrome de Wolff Parkinson White en Atención Primaria
}

\author{
S. del Castillo ArRojo, L. Sierra Santos* \\ Cardióloga. Hospital Gregorio Marañón. Madrid. \\ *Médico de Familia. C.S. Manzanares el Real. Madrid
}

\section{RESUMEN}

El síndrome de Wolff Parkinson White es una anomalía cardiaca congénita que afecta a un 0,1-3\%o de la población general. Se diagnostica de síndrome de Wolff Parkinson White a aquellos pacientes con preexcitación en el electrocardiograma e historia clínica de palpitaciones.

La presentación clínica más habitual son episodios paroxísticos de palpitaciones, de carácter benigno, aunque en estos pacientes la probabilidad de muerte súbita es superior a la población general.

El tratamiento de estos pacientes se ha modificado enormemente en los últimos años gracias al desarrollo de la ablación con radiofrecuencia, tratamiento definitivo con alto porcentaje de éxito y algunos riesgos.

El diagnóstico debe realizarse por el médico de Atención Primaria, así como la derivación al cardiólogo de los casos especiales que puedan precisar pruebas diagnósticas complejas y de aquéllos que por su sintomatología o por su riesgo (ya sea por motivos médicos o laborales), precisan el tratamiento definitivo de la ablación con radiofrecuencia.

Palabras clave: Síndrome de Wolf Parkinson White. Taquiarritmias. Ablación con radiofrecuencia. Vías cardiacas accesorias.
Management and diagnosis of Wolff Parkinson White syndrome by Primary Health Care

Key words: Wolff Parkinson White syndrome. Arrythmia. Pre-excitacion syndrome. Catheter ablation.

\section{INTRODUCCIÓN}

El síndrome de Wolff Parkinson White (WPW) es una anormalidad cardiaca congénita que consiste en la presencia de un haz anómalo (haz de Kent) que salva el sistema normal de conducción uniendo directamente aurículas y ventrículos. Se trata de un síndrome de preexcitación, es decir, de un trastorno 


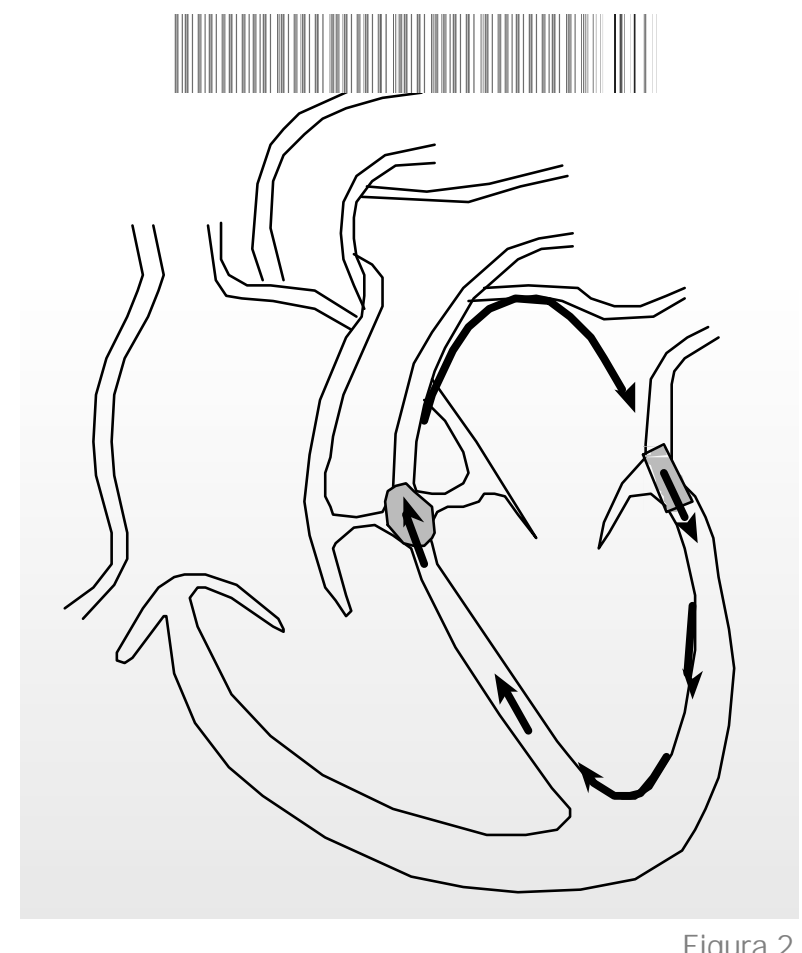

Esquema de la reentrada antidrómica

otros desencadenantes ${ }^{2}$. Su duración es variable, de unos minutos a horas. El mecanismo que más frecuentemente la provoca en estos pacientes es la reentrada ortodrómica (Fig. 1). Se produce un circuito de reentrada en la cual el impulso se conduce de las aurículas a los ventrículos exclusivamente por el nodo AV y sube de los ventrículos a las aurículas por la vía accesoria. Por este motivo la taquicardia es de QRS estrecho y sin datos de preexcitación. Aproximadamente un $60 \%$ de las taquicardias en el síndrome de WPW tienen este mecanismo. Mucho más infrecuente (entre un 1 y un $11 \%$ según las series) es la reentrada antidrómica. El impulso baja por la vía accesoria en este caso y sube de los ventrículos a las aurículas por el nodo AV (Fig. 2) ${ }^{16-18}$. Por ello el electrocardiograma en taquicardia mostrará un QRS ancho, con preexcitación máxima. La segunda taquicardia en cuanto a frecuencia de presentación es la fibrilación auricular (FA). La presentan entre un 12 y un $35 \%$ de los pacientes con síndrome de WPW $^{19,20}$. Las vías accesorias, y más si son múltiples, favorecen la aparición de FA. Esta arritmia se puede presentar como una taquicardia de QRS estrecho o de QRS ancho, bien sea por preexcitación (conducción por la vía accesoria) o bien por aberrancia o bloqueo de rama en el haz de $\mathrm{His}^{19,20 .}$ La FA conducida a los ventrículos a través de una vía accesoria puede desencadenar, si la respuesta ventricular es rápida, síncope e incluso fibrila- ción ventricular y muerte súbita ${ }^{21,22}$. Un porcentaje no despreciable de pacientes con síndrome de WPW presentan síncope. En un subgrupo de ellos el síncope representa la presencia de arritmias con respuesta ventricular muy rápida y mal tolerada, pero en otros se ha achacado a una anormal respuesta vasomotora durante la taquicardia ${ }^{1,23}$. Además el síncope puede estar o no en relación con un episodio de taquicardia (recordemos que se trata con frecuencia de pacientes jóvenes, población con una relativamente alta incidencia de síncope vaso-vagal ${ }^{24}$. De hecho los estudios no han demostrado un valor pronóstico del síncope en estos pacientes. La muerte súbita puede ser también una forma de debut de la enfermedad aunque el riesgo de muerte súbita en pacientes asintomáticos es menor del $1 / 1000^{10,25,26}$. Se considera que la mayor parte de episodios de muerte súbita en pacientes con WPW está en relación con episodios de FA con conducción al ventrículo por la vía accesoria y posterior degeneración a fibrilación ventricular. La presencia de intervalos RR menores de 250 mseg durante la FA o taquicardias preexcitadas espontáneas es el más importante marcador de riesgo de muerte súbita por fibrilación ventricular. En cambio, el mismo dato durante FA inducida durante el estudio electrofisiológico tiene una especificidad muy baja, lo que ha influido en el abandono por parte de la mayor parte de los expertos del estudio electrofisiológico en pacientes asintomáticos ${ }^{27,28}$. También es mayor el riesgo en pacientes con antecedentes familiares de muerte súbita y con múltiples vías accesorias ${ }^{29}$. En un estudio realizado por Atié se demostraba que los pacientes con taquicardia por reentrada antidrómica tenían un mayor riesgo de muerte súbita por degeneración de FA preexcitada en fibrilación ventricular. Además estos pacientes tienden a debutar a edades tempranas y con frecuencia presentan síncope o presíncope en su evolución ${ }^{3,18}$.

\section{DIAGNÓSTICO}

Como ya hemos comentado el diagnóstico del Síndrome de WPW es clínico y electrocardiográfico. Podemos encontramos ante un paciente que acude por uno o varios episodios de palpitaciones o pacientes en los que se realiza un electrocardiograma por otro motivo y se descubre la anomalía electrocardiográfíca. En la historia clínica se debe hacer incapié en la presencia de dos síntomas claves:

1. Episodios paroxísticos de palpitaciones. Es importante definir las características de los epi- 
gramas seriados ${ }^{10}$.

La radiografía de tórax es normal en los pacientes sin patologías asociadas.

Se debe realizar un ecocardiograma a todos los pacientes sintomáticos o con alteraciones en la exploración física. Recomendamos también realizar ecocardiograma a todos los pacientes diagnosticados en edades tempranas por la asociación del síndrome con malformaciones cardiacas congénitas.

En casos seleccionados estará indicado el holter de arritmias en pacientes con alta sospecha diagnóstica del WPW en los que no se haya podido registrar la taquicardia. En los raros casos en los que la taquicardia se relaciona con el ejercicio físico estaría justificada la prueba de esfuerzo.

\section{TRATAMIENTO MÉDICO}

\section{Tratamiento crónico}

Los pacientes que presentan la anormalidad electrocardiográfica del síndrome de WPW pero están asintomáticos no precisan tratamiento alguno $^{31}$. Un caso especial lo constituyen ciertas profesiones de riesgo en las que se excluye al personal con datos electrocardiográficos de WPW. Así ocurre en nuestro país con pilotos y deportistas de élite. El bajísimo riesgo de muerte súbita en estos pacientes por sí mismo no justifica la realización del estudio electrofisiológico y la ablación, aunque sí se puede plantear por exigencia laboral explicando al paciente los riesgos de la intervención ${ }^{25}$.

En pacientes clínicamente sintomáticos debemos distinguir mediante la historia clínica cuidadosa, la frecuencia, duración y número de episodios de las taquicardias:

- Los pacientes con episodios infrecuentes de taquicardias, bien toleradas, no precisan ningún tratamiento crónico. Se recomienda enseñarles a realizar las maniobras vagales o de Valsalva y en caso de que con ellas no ceda la taquicardia deberán acudir a un centro de urgencias para su tratamiento agudo. Deben seguir revisiones periódicas por su médico de cabecera o cardiólogo para evaluar posibles cambios en la sintomatología ${ }^{30,31}$.

- Los pacientes con episodios frecuentes de taquicardias o que toleren hemodinámicamente mal las mismas deben ser remitidos al cardiólogo, quien valorará de acuerdo con las preferencias del paciente el manejo farmacológico o la indicación de ablación con radiofrecuencia. En general, existe una tendencia global y una recomendación por las
Tabla I

INDICACIONES DEL ESTUDIO ELECTROFISIOLÓGICO

Y DE LA ABLACIÓN CON RADIOFRECUENCIA EN

PACIENTES CON SÍNDROME DE PREEXCITACIÓN DE LA SOCIEDAD ESPAÑOLA DE CARDIOLOGÍA

CLASE

(acuerdo general de su eficacia y utilidad)

Pacientes sintomáticos por taquicardias por reentrada aurículo-ventricular o taquiarrítmias auriculares conducidas por la vía.

Ablación en:

1. Preferencia del paciente.

2. Arritmias resistentes a medicación.

3. Intolerancia a la medicación.

\section{CLASE Ila}

(la mayoría de evidencia y opiniones están a favor de su eficacia y utilidad)

1. Pacientes poco sintomáticos, con evidencias clínicas de mala o nula conductividad anterógrada de la vía accesoria, que presenten intolerancia a la medicación 0 prefieran la ablación.

2. Pacientes asintomáticos con preexcitación en los que la presencia de ésta suponga un obstáculo para el desarrollo de su vida habitual (profesión, práctica deportiva habitual, seguros de vida, etc.), una afectación de la calidad de vida o tengan profesiones de riesgo.

3. Pacientes con cardiopatía y/o con riesgo conocido de padecer taquiarrítmias auriculares.

\section{CLASE IIb}

(eficacia menos establecida)

1. Pacientes asintomáticos.

\section{CLASE III}

(procedimientos no eficaces e incluso perjudiciales)

1. Pacientes con arritmias mediadas por la vía accesoria, controlables con medicación y que prefieran ésta a la ablación.

2. Pacientes asintomáticos con evidencias clínicas de mala conductividad anterógrada a través de la vía accesoria (ablación cosmética).

Sociedades Españolas (Tabla I) y Americana de Cardiología de aumentar las indicaciones de la ablación por radiofrecuencia por ser en el momento actual curativa en un porcentaje del $95 \%$ con un 
venoso con adenosina o ATP o con verapamilo. Se considera de primera elección la adenosina o ATP por su seguridad y cortísima vida media. Es importante asegurarse de que se está administrando la dosis correcta (en el caso de adenosina $6 \mathrm{mg}$ y si no cede una segunda dosis de $12 \mathrm{mg}$. Para el ATP, $10 \mathrm{mg}, 20 \mathrm{mg}$ y hasta $40 \mathrm{mg}$ ) y administrarla en bolo muy rápido seguido de suero fisiológi$\mathrm{co}^{38,39}$. Este fármaco se puede utilizar en todos los pacientes que no tengan antecedentes de asma o broncoespasmo, incluso en pacientes con disfunción ventricular. Produce efectos secundarios en casi un $100 \%$ de los pacientes en forma de opresión torácica, tos y calor facial, lo que se debe advertir al paciente siempre antes de su uso, resaltando que no durará más de un minuto y que se trata de una respuesta normal al fármaco y no una alergia al mismo.

La efectividad del ATP o adenosina para revertir taquicardias por reentrada que incluya el nodo AV (es decir, las del WPW, vía accesoria oculta y las aún más frecuentes taquicardias intranodales) es cercana al 95\%. Este fármaco es especialmente útil en centros de Atención Primaria distantes del hospital de referencia por su eficacia, seguridad y facilidad de uso. En pacientes con WPW a los que se administró ATP por una taquicardia supraventricular se ha descrito que hasta el $12 \%$ pasan a FA y en casos aislados a FA rápida con mala tolerancia clínica, por lo que se aconseja tener un desfibrilador cerca en el momento de la administración ${ }^{3}$.

En el caso de presentar contraindicaciones para el uso del ATP se puede administrar una ampolla de verapamilo intravenosa $(5 \mathrm{mg})$ en dos minutos. Este fármaco tiene la ventaja de carecer de los efectos desagradables del ATP o la adenosina y de ejercer un efecto más prolongado por lo que puede prevenir las recurrencias inmediatas. Su desventaja es que tiene un efecto inotrópico negativo, por lo que está contraindi cado en pacientes con disfunción ventricular izquierda ${ }^{1}$.

Un porcentaje escaso de pacientes se presentará con una taquicardia regular de QRS ancho. Ante cualquier paciente, de cualquier edad o patología previa que presente una taquicardia de QRS ancho se debe actuar con la máxima prudencia. En este grupo de pacientes la existencia de QRS ancho se puede deber a un bloqueo de rama preexistente, aberrancia de rama o a una taquicardia antidrómica (es decir, que baje de la aurícula al ventrículo por la vía accesoria y suba por el nodo AV). Pero jamás se debe olvidar que el tener un síndrome de WPW no excluye a un paciente de padecer una taquicardia ventricular, que debe ser nuestra hipótesis diagnóstica primera siempre que veamos una taquicardia con QRS an- cho. Por lo tanto, si la taquicardia es mal tolerada se recomienda cardioversión eléctrica inmediata y si es bien tolerada se debe remitir a un centro hospitalario en un transporte monitorizado pudiéndose realizar un intento diagnóstico-terapéutico con ATP o adenosina si no hay contraindicaciones. En el caso de ser una taquicardia que incluya el nodo AV en la reentrada, provocará el cese brusco de la arritmia; si es una taquicardia ventricular no afectará a la misma y si es una taquicardia puramente auricular producirá un bloqueo AV transitorio que nos permitirá ver la actividad auricular ${ }^{40}$.

No se recomienda el verapamilo en las taquicardias de QRS ancho por la posibilidad de tratarse de una taquicardia ventricular ${ }^{1}$.

En los pacientes con taquicardia irregular de QRS ancho, la primera sospecha diagnóstica es una fibrilación auricular preexcitada. Se recomienda monitorizar y transladar a un centro hospitalario y, si es mal tolerada, cardioversión inmediata. Es necesario recalcar en este punto que la digital y el verapamilo están formalmente contraindicados en estas taquicardias por la posibilidad de incrementar la conducción por la vía anómala y provocar un deterioro hemodinámico e incluso una fibrilación ventricular.

\section{PROCEDIMIENTOS INVASIVOS: CIRUGÍA, ESTUDIO ELECTROFISIOLÓGICO Y ABLACIÓN CON RADIOFRECUENCIA}

Desde finales de los años sesenta se utiliza el tratamiento quirúrgico para interrumpir las vías accesorias en pacientes con el síndrome de WPW no controlables con medicación. Tras el inicio y generalización de la ablación con radiofrecuencia la cirugía se ha relegado sólo a casos con síntomas intratables en los que no ha sido posible la ablación percutánea o pacientes que precisan cirugía cardiaca por otro motivo (por ejemplo en la cirugía de la malformación de Ebstein) ${ }^{41}$.

En pacientes seleccionados por su alto riesgo de muerte súbita, frecuencia de los episodios de taquicardia, mal control con medicación e incluso la propia preferencia del paciente que no desee tomar fármacos a largo plazo, está indicada la realización de un estudio electrofisiológico. La técnica consiste en la introducción de catéteres en las cavidades cardiacas con control radioscópico, generalmente por vía femoral, y estimulación eléctrica para valorar o confirmar la existencia de una o más vías accesorias, sus propiedades de conducción y su localización; para en el mismo procedimiento realizar la ablación con radiofrecuencia. 
JP, et al. Catheter ablation of accesory pathways, atrioventricular nodal reentrant tachycardia, and the atrioventricular junction. Final results of a prospective, multicenter clinical trial. Circulation 1999; 99: 262-70.

6. Jackman W, Xunzhang W, Friday KJ, Roman CA, Moulton KP, Beckman KJ, et al. Catheter ablation of accessory atrioventricular pathways (Wolff Parkinson White Sindrome) by radiofrecuency current. N Engl J Med 1991; 324: 1605-11.

7. Calkins H, Langberg J, Sousa J, El-Atassi R, Leon A, Kou $\mathrm{W}$, et al. Radiofrecuency catheter ablation of accesory atrioventricular connections in 250 patients. Circulation 1992; 85: 1337-46.

8. Kovoor P, Ricciardello M, Collins L, Uther JB, Ross DL. Risk to patients from radiation associateds with radiofrecuency ablation for supraventricular tachycardia. Circulation 1998; 98: 1534-40.

9. Viñolas Prat X, Rodriguez Font E, Alonso Martín C, Bayés de Luna A. Síndromes arritmológicos. Medicine (Idepsa) 1996; 7(20): 805-10.

10. Munger TM, Packer DL, Hammill SC, Feldman BJ, Bailey $\mathrm{KR}$, Ballard DJ, et al. A population study of the natural history of Wolff-Parkinson-White Syndrome in Olmsted County, Minessota, 1953-1989. Circulation 1993; 87: 866-73.

11. Viñolas Prat X, Rodriguez Font E, Alegret Colome JM, Bayés de Luna A. Arritmias hiperactivas supraventriculares. Medicine (Idepsa) 1996; 7(19): 762-70.

12. Castellano Reyes C, Pérez de Juan Romero MA, Espinosa Caliani JS. Síndromes de preexcitación. En: Castellanos Reyes C, Pérez de Juan Romero MA. Electrocardiografía Clínica. Madrid: Ediciones Harcourt, 2000; 81-100.

13. López Minguez JR, Urbano Galvez GM, Gonzalez Fernandez R, García Andoain JM, Cimbora Ortega A, Millan Nuñez V, et al. Fibrilación auricular y el Síndrome de Wolff-Parkinson-White en un paciente de 68 años con esclerosis tuberosa. Rev Esp Card 1999; 52(3): 207-10.

14. Chen SA, Chiang CE, Tai CT, Lee SH, Chiou CW, Ueng $\mathrm{KC}$, et al. Longitudinal clinical and electrophysilogical assessment of patients with symptomatic Wolff-ParkinsonWhite Syndrome and atrioventricular node reentrant tachycardia. Circulation 1996; 93: 2023-32.

15. Deal BJ, Keana JF, Gillette PC, Garson A Jr. Wolff-Parkinson-White syndrome and supraventricular tachycardia during infancy: Management and follow-up. J Am Coll Cardiol 1985; 5(1):130-5.

16. Packer DL, Gallagher JJ, Prystowsky EN. Physiologic substrate for antidromic reciprocating tachycardia: prerequisite characteristics of the accessory pathway and AV conduction system. Circulation 1992; 85(2): 574-88.

17. Bardy GH, Packer DL, German LD, Gallagher JJ. Preexcited reciprocating tachycardia in patients with Wolff-Parkinson-White Syndrome: incidence and mechanisms. Circulation 1984; 70(3): 377-91.

18. Atie J, Brugada P, Brugada J, Smeets JL, Cruz FS, Peres A, et al. Clinical and electrophysiologic characteristics of patients with antidromic circus movement tachycardia in the Wolff-Parkinson-White Syndrome. Am J Cardiol 1990; 66(15):1082-91

19. Wellens HJ, Durrer D. Wolff-Parkinson-White Syndrome and atrial fibrillation. Am J Cardiol 1974; 34(7): $777-82$.

20. Iesaka Y, Yamane T, Takahashi A, Goya M, Kojima S, Soejima Y, et al. Retrograde multiple and multifiber accessory pathway conduction in the Wolff-Parkinson-White Syndrome: potential Precipitating factor of atrial fibrillation. J Cardiovasc Electrophysiol 1998; 9: 141-51.
21. Teo WS, Klein GJ, Guiraudon GM, Yee R, Leitch JW, McLellan D, et al. Multiple accessory pathways in the Wolff-Parkinson-White Syndrome as a risk factor for ventricular fibrillation. Am J Cardiol 1991; 68: 889-91.

22. Montoya PT, on behalf of the European Registry of sudden death in the Wolff-Parkinson-White. Ventricular fibrillation in the Wolff-Parkinson-White Syndrome (Abstract). Circulation 1988; 78(11): 11-22.

23. Leitch JW, Klein GJ, Yee R, Leather RA, Kim YH. Syncope associated with supraventicular tachycardia rate or vasomotor response? Circulation 1992; 85(3): 1064-71.

24. Kapoor WN. Syncope. N Eng J Med 2000; 343(25): 1856-62.

25. Epstein AE, Miles WM, Benditt DG, Camm AJ, Darling EJ, Friedman PL, et al. Personal and public safety issues related to arrythmias that may affect consciousness: implications for regulation and physician recomendations. A medical/scientific statement from the AHA and the NASPE. Circulation 1996; 94: 1147-66.

26. Klein GJ, Prystowsky EN, Yee R, Sharma AD, Laupacis A. Asymptomatic Wolff-Parkinson-White: should we intervene? Circulation 1989; 80: 1902-5.

27. García Civera R. Electrofisiología de las vías accesorias AV comunes. En: García Civera R, Ruiz Granell R, Morell Cabedo S, Sanjuán Máñez R, Martínez Leon J, Botella Solana $\mathrm{S}$, et al. Electrofisiología Cardíaca Clínica y Ablación. Madrid: McGraw-Hill-Interamericana, 1999; 181-203.

28. Leitch JW, Klein GJ, Yee R, Murdok C. Prognostic value of electrophysiology testing in asymptomatic patients with Wolff-Parkinson-White pattern. Circulation 1990; 82 (5):1718-23

29. Iesaka Y, Yamane T, Takahashi A, Goya M, Kojima S, Soejima Y, et al. Retrograde multiple and multifiber accesory pathway conduction in the Wolff-Parkinson-White syndrome: potential precipitating factor of atrial fibrillation. J Cardiovasc Electrophysiol 1998; 9: 141-51.

30. Zimetbaum P, Josephson ME. Evaluation of patients with palpitations. N Engl J Med 1998; 338: 1369-73.

31. Almendral Garrote J, Marín Huerta E, Medina Moreno O, Peinado Peinado R, Pérez Álvarez L, Ruiz Granell R, et al. Guías de práctica clínica de la Sociedad Española de Cardiología en arritmias cardíacas. Rev Esp Cardiol 2001; 54: 307-67.

32. Zipes DP, DiMarco JP, Gillette PC, Jackman WM, Myerburg RJ, Rahimtoola SH, et al. Guidelines for Clinical Intracardiac Electrophysiological and Catherter Ablation Procedures. A Report of the American College of Cardiolgy/ American Heart Association Task Force on Practice Guidelines. Circulation 1995; 92: 673-91.

33. Goldschlager N, Goldman MJ. Síndromes de preexcitación. En: Goldschlager N, Goldman MJ. Principios de electrocardiografía clínica. $13^{\mathrm{a}}$ ed. Méjico DF: El manual moderno, 1991; 245-56.

34. Chou TC. Wolff-Parkinson-White Syndrome and its variants. En: Chou TC. Electrocardiography in clinical practice. $3^{\text {rd }}$ ed. Philadelphia: WB Saunders Company, 1991; 429-58.

35. Bubien RS, Knotts-Dolson SM, Plumb VJ, Kay GN. Effect of Radiofrecuency Catheter Ablation on HealthRelated Quality of Life and Activities of Daily Living in Patients With Recurrent Arrhythmias. Circulation 1996; 94: 1585-91.

36. Echt DS, Liebson PR Mitchell LB, Peters RW, ObiasManno D, Barker AH, et al. Mortality and morbidity in patients receiving encainide, flecainide or placebo: The Cardiac Arrhythmia Suppression Trial. N Engl J Med 1991; 324 (12): 781-8.

37. Pritchett ELC, Wilkinson WE, Clair WK, McCaurthy EA Comparison of mortality in patients treated with propafenone 